\title{
Solitary Pulmonary Nodule Due to Mycobacterium kansasii
}

\author{
Masaaki Abe ${ }^{1}$, Yoshihiro Kobashi ${ }^{1}$, Keiji Mouri ${ }^{1}$, Yasushi Obase ${ }^{1}$, Naoyuki Miyashita ${ }^{1}$, \\ Masao Nakata ${ }^{2}$ and Mikio Oka ${ }^{1}$
}

\begin{abstract}
A 46-year-old man with no past history of underlying disease was admitted to our hospital for examination of abnormal chest shadow on chest radiograph. He had no respiratory symptoms on admission. Chest radiograph showed a solitary nodule $(35 \times 20 \mathrm{~mm})$ in the left upper lung field. On chest $\mathrm{CT}$, this nodule in the left upper lobe $\left(\mathrm{S}^{1+2}\right)$ did not demonstrate calcification, the margin was clear but irregular, and there was pleural indentation. The solitary nodule was strongly positive on PET/CT. Therefore, we suspected primary lung cancer. Because we could not establish the diagnosis by bronchoscopic examination, video-assisted thoracoscopic surgery (VATS) was performed. Histological diagnosis of the solitary nodule demonstrated epitheloid granuloma with caseous necrosis. Smear test of the resected tissue was positive for acid-fast bacilli and culture was positive for mycobacteria, which was identified as Mycobacterium kansasii. There are a few case reports of solitary nodule due to $M$. kansasii.
\end{abstract}

Key words: solitary nodule, Mycobacterium kansasii

(Intern Med 50: 775-778, 2011)

(DOI: 10.2169/internalmedicine.50.4958)

\section{Introduction}

Mycobacterium kansasii is the second most common nontuberculous mycobacteria (NTM) following Mycobacterium avium complex in the United states and Japan $(1,2)$. The frequency of isolation of $M$. kansasii has been increasing recently (2). The radiological findings of pulmonary M. kansasii disease are nearly identical to those of pulmonary tuberculosis. Although cavitation was found in $90 \%$ of patients with pulmonary $M$. kansasii disease in older reports $(3,4)$, this finding was observed in $30 \sim 50 \%$ of patients with pulmonary $M$. kansasii disease in recent reports $(5,6)$. A variety of radiological findings have been reported in pulmonary $M$. kansasii disease including noncavitary or nodular/bronchiectatic lesions $(6,7)$. However, in a search of the literature we found only one report of pulmonary $M$. kansasii disease with a solitary nodule (8). In the present case, it was difficult to distinguish lung cancer because PET/CT was positive for solitary pulmonary nodule. Finally, we obtained a pathological diagnosis of pulmonary $M$. kansasii disease causing a solitary nodule after surgical resection of the nodule.

\section{Case Report}

A 46-year-old man with no past history of underlying disease was admitted to our hospital in mid-August 2010 because of an abnormal chest shadow caused by a solitary nodule with a comparatively clear margin that had recently appeared in the left $\mathrm{S}^{1+2}$. He had no respiratory symptoms on admission. There were no abnormal findings on chest radiograph obtained the previous year.

On admission, there were no abnormal physical or laboratory findings (Table 1). Although he had a past history of Bacille Calmette Guérin (BCG) vaccination, purified protein derivatives (PPD) was negative and QuantiFERON Gold test also showed a negative response.

Chest radiograph on admission showed a solitary nodule $(25 \times 20 \mathrm{~mm})$ in the left upper lung field (Fig. 1). Chest computed tomography (CT) disclosed a solitary nodule with a comparatively clear margin and pleural indentation, but without cavity, calcification, satellite lesion (Fig. 2). PET/CT demonstrated a hot lesion corresponding to this solitary nod-

${ }^{1}$ Division of Respiratory Disease, Department of Medicine, Kawasaki Medical School, Japan and ${ }^{2}$ Department of Thoracic Surgery, Kawasaki Medical School, Japan

Received for publication December 7, 2010; Accepted for publication January 6, 2011

Correspondence to Dr. Yoshihiro Kobashi, yoshihiro@med.kawasaki-m.ac.jp 
Table 1. Laboratory Data on Admission

\begin{tabular}{lllc}
\hline $\begin{array}{l}\text { Peripheral blood } \\
\text { RBC }\end{array}$ & $502 \times 10^{4} / \mu \mathrm{L}$ & CRP & $<0.03 \mathrm{mg} / \mathrm{dL}$ \\
Hb & $15.8 \mathrm{~g} / \mathrm{dL}$ & $\mathrm{Na}$ & $140 \mathrm{mEq} / \mathrm{L}$ \\
$\mathrm{Ht}$ & $47.7 \%$ & $\mathrm{~K}$ & $4.4 \mathrm{mEq} / \mathrm{L}$ \\
WBC & $5760 / \mu \mathrm{L}$ & $\mathrm{Cl}$ & $103 \mathrm{mEq} / \mathrm{L}$ \\
Plate & $17.2 \times 10^{4} / \mu \mathrm{L}$ & Serology & \\
ESR & $18 \mathrm{~mm} / \mathrm{hr}$ & $\beta-$ D-glucan & $<6.0 \mathrm{pg} / \mathrm{mL}$ \\
Chemical screening & & Cryptococcus antigen & $(-)$ \\
TP & $7.4 \mathrm{~g} / \mathrm{dL}$ & Aspergillus antigen & $(-)$ \\
Alb & $4.6 \mathrm{~g} / \mathrm{dL}$ & Tumor marker & \\
ALT & $13 \mathrm{U} / \mathrm{L}$ & CEA & $2.2 \mathrm{ng} / \mathrm{mL}$ \\
AST & $18 \mathrm{U} / \mathrm{L}$ & SLX & $17.1 \mathrm{U} / \mathrm{mL}$ \\
Bil(T) & $0.7 \mathrm{mg} / \mathrm{dL}$ & CYFRA & $<1.0 \mathrm{ng} / \mathrm{mL}$ \\
ALP & $232 \mathrm{U} / \mathrm{L}$ & SCC & $1.5 \mathrm{ng} / \mathrm{mL}$ \\
LDH & $128 \mathrm{U} / \mathrm{L}$ & ProGRP & $28.3 \mathrm{pg} / \mathrm{mL}$ \\
ChE & $384 \mathrm{U} / \mathrm{L}$ & NSE & $8.1 \mathrm{ng} / \mathrm{mL}$ \\
Crn & $0.66 \mathrm{mg} / \mathrm{dL}$ & PPD & $0 \times 0 \mathrm{~mm}$ \\
BUN & $11 \mathrm{mg} / \mathrm{dL}$ & QuantiFERON TB-Gold & $<0.05$ \\
UrA & $4.6 \mathrm{mg} / \mathrm{dL}$ & ESAT-6 & $<0.05$ \\
\hline
\end{tabular}

PPD: purified protein derivatives (diameter of induration)

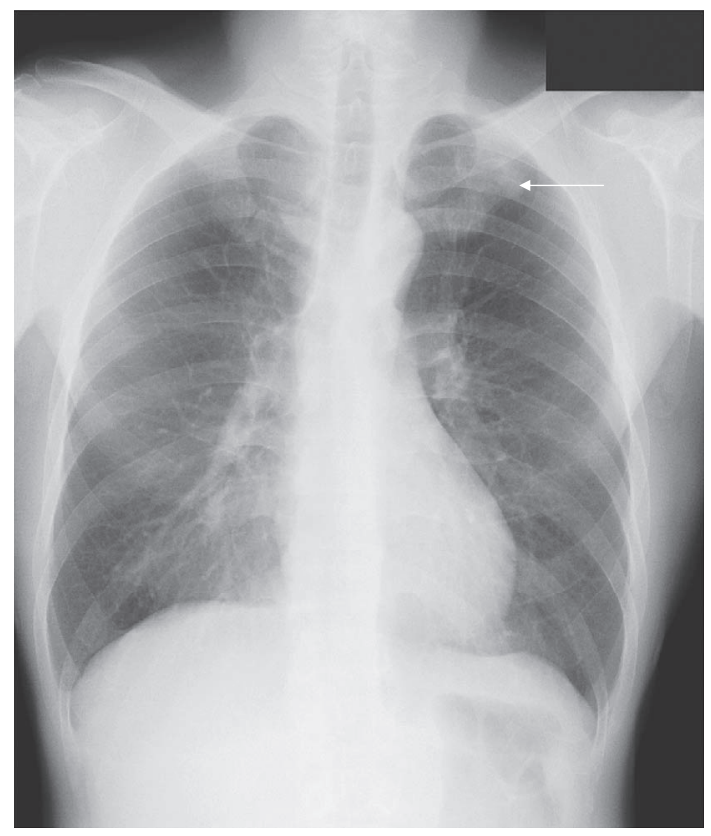

Figure 1. Chest radiograph showed a solitary nodule $(25 \times 20 \mathrm{~mm})$ in the left upper lung field $(\rightarrow)$.

ule, but there were no other hot lesions (Fig. 3). We could not establish the definite diagnosis by bronchoscopic examination. Therefore, we performed partial resection by videoassisted thoracoscopic surgery (VATS) to deny the possibility of lung cancer.

The histological findings on VATS showed an epitheloid granuloma with caseating necrosis (Fig. 4). Smear test of the resected tissue was positive for fast-acid bacilli and the

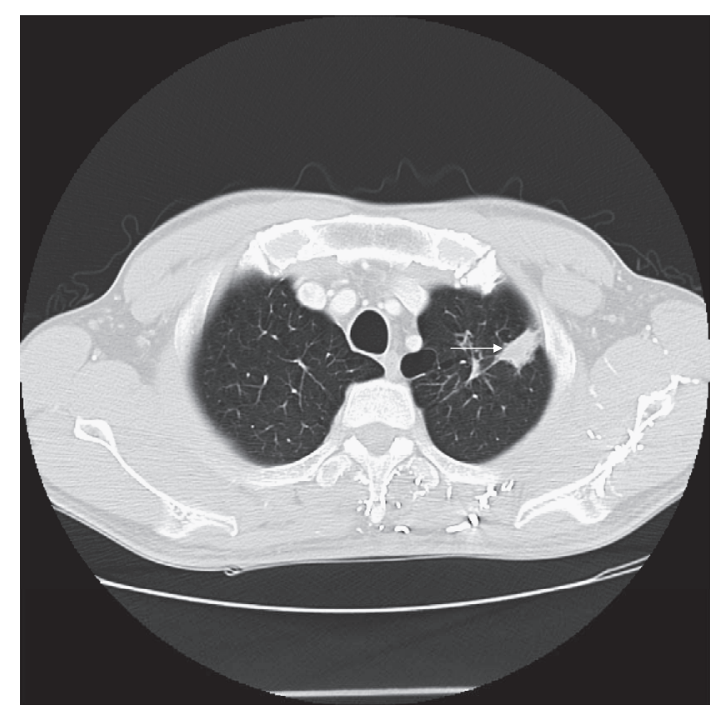

Figure 2. Chest computed tomography (CT) showed a solitary nodule $(\rightarrow)$ with a comparatively clear margin and pleural indentation in segment $\mathrm{S}^{1+2}$ of the left upper lobe.

culture test was also positive. The final diagnosis due to DNA-DNA hybridization (DDH) test was pulmonary $M$. kansasii disease (solitary nodule type).

Although antituberculous drugs were not administered after partial resection of the left upper lobe by VATS, there has not been any relapse in this patient.

\section{Discussion}

In Japan, M. kansasii constitutes $15-20 \%$ of NTM organ- 


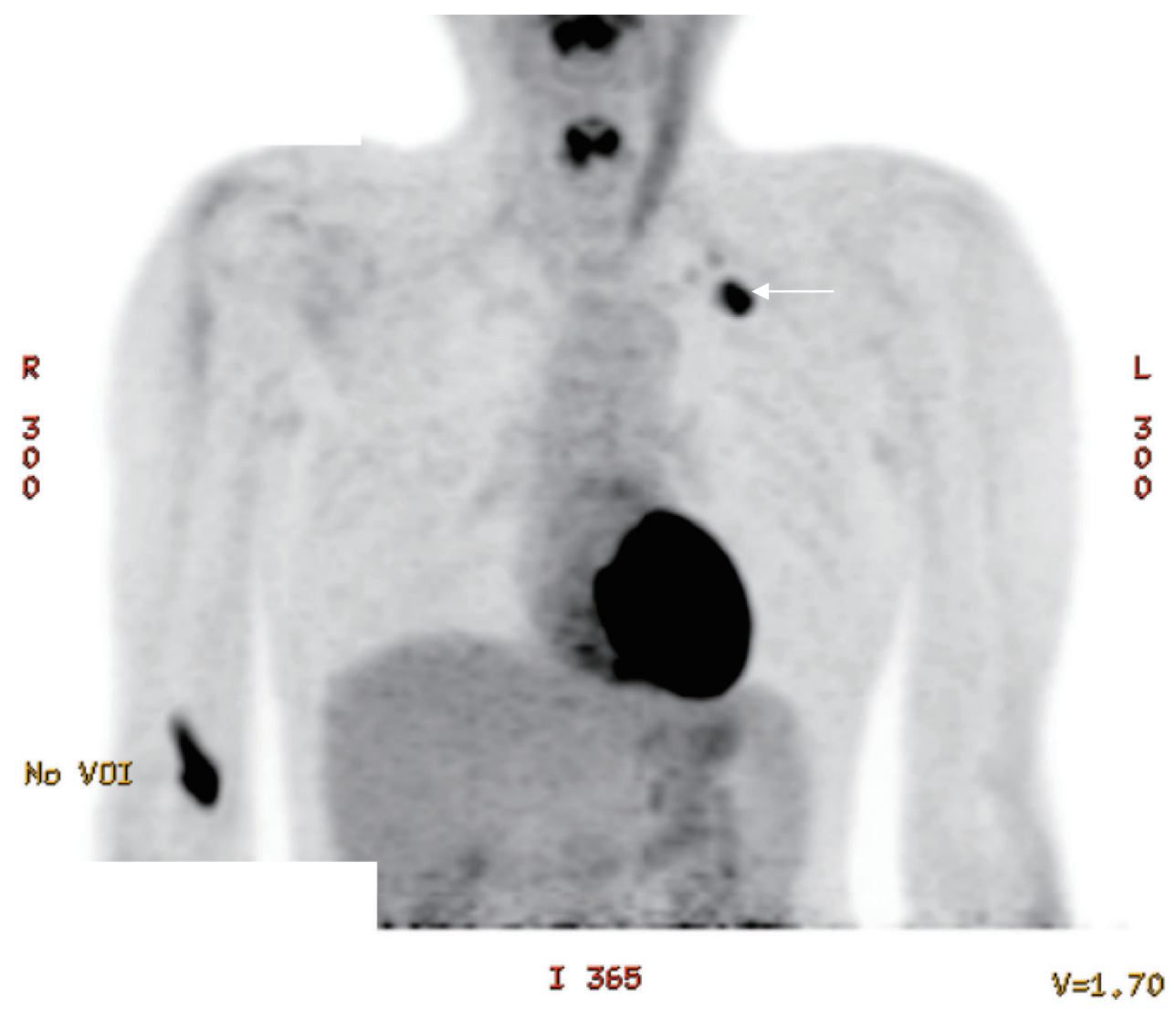

Figure 3. PET/CT showed a hot lesion corresponding to this solitary nodule $(\rightarrow)$, but there were no other hot lesions.

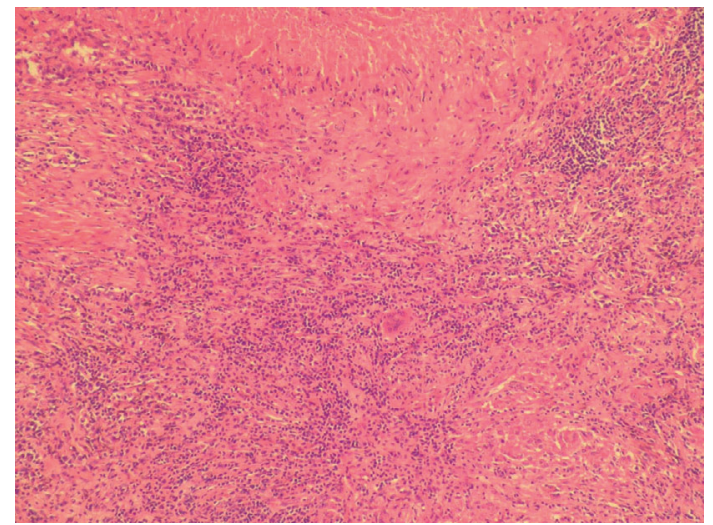

Figure 4. Histological findings showed an epitheloid granuloma with caseating necrosis (Hematoxylin and Eosin staining, $\times 100)$

isms isolated from clinical specimens (2). The findings on the chest radiograph of pulmonary $M$. kansasii disease are similar to those of pulmonary tuberculosis, including cavitary infiltrates with upper lobe predilection. However, noncavitary lung disease has also recently been recognized as part of the spectrum of pulmonary $M$. kansasii disease $(5,6)$. We encountered a peculiar case of solitary nodule formation due to M. kansasii in the present patient. The most common cause of benign granulomas is pulmonary tuberculoma. Pulmonary tuberculomas are pathologically diagnosed by transbronchial lung biopsy or computed tomogra- phy (CT)-guided lung biopsy in most cases. In some cases, surgical lung biopsy is performed and solitary pulmonary nodules similar to granulomas are assumed to be attributable to $M$. tuberculosis infection. In these cases, bacteriological confirmation is not always performed $(9,10)$. Therefore, there was no distinction made between tuberculous and NTM infections $(11,12)$. The possibility is high that cases of solitary pulmonary nodules due to NTM infection may have been included among with pulmonary tuberculoma.

Gribetz et al first reported 20 cases of solitary pulmonary nodules due to NTM infection in 1982 (13). Twelve of 20 cases were due to Mycobacterium avium complex (MAC) infection and there were no cases due to M. kansasii. Although Hahm et al and Yonemori et al reported cases of solitary pulmonary nodules due to NTM infection (16 cases and 24 cases, respectively) $(14,15)$, there has been only one case of solitary pulmonary nodule due to $M$. kansasii diagnosed by thoracotomy as reported by Kurasawa et al (8).

Diseases showing a solitary pulmonary nodule on radiography consist of primary or metastatic lung cancer, benign tumor of the lung, inflammatory granuloma of the lung (16). Although radiological diagnostic methods have been rapidly developing since the introduction of CT, it is difficult to establish a differential diagnosis for solitary pulmonary nodules $<3 \mathrm{~cm}$ (17). In the present case, because the solitary pulmonary nodule had a comparatively clear margin and pleural indentation, but did not demonstrate calcification or satellite lesions, we could not deny the possibility of lung 
cancer and performed partial resection by VATS. In addition, the solitary pulmonary nodule showed high FDG uptake on FDG-PET imaging. FDG-PET is capable of demonstrating the glucose metabolism of a solitary pulmonary nodule, indicating an active lesion regardless of whether the lesion is benign or malignant. Granulomatous nodules such as tuberculoma frequently show positive results $(10,18)$. FDG-PET is obviously not a test of malignancy but of metabolism. This is also true for solitary pulmonary nodule due to NTM infection (19). Because the present case also demonstrated that a solitary pulmonary nodule due to $M$. kansasii can exhibit high FDG uptake on FDG-PET imaging, it was difficult to distinguish lung cancer.

Another potential tool supporting the diagnosis of pulmonary $M$. kansasii disease is interferon-gamma release assays (IGRAs). Although patients with pulmonary infection due to M. kansasii, which demonstrate $M$. tuberculosis-specific antigens such as CFP-10 or ESAT-6, are expected to present a positive response, about $50 \%$ of patients with M. kansasii infection showed positive IGRAs test results in our previous study (20). We think that it is difficult for IGRAs to become a supportive diagnostic method for pulmonary M. kansasii disease since the present case showed a negative response on QFT Gold test.

Concerning the management of patients with solitary pulmonary nodule due to M. kansasii infection, there is no evidence indicating whether anti-M. kansasii antibiotic treatment should be performed after surgical resection of solitary pulmonary nodule as in other mycobacterial diseases. Although we did not perform combined chemotherapy after the surgical resection for the present case, we think we must continue to monitor this patient for relapse in the future.

In conclusion, although lung cancer or pulmonary tuberculoma is first suspected when a solitary nodule is strongly positive on PET/CT such as in the present case, NTM infection including MAC or M. kansasii infection must also be considered as differential diagnoses of solitary pulmonary nodule. Because the treatment method differs based on the disease causing the solitary pulmonary nodule, it is important to distinguish malignant and benign diseases by performing surgical resection and to identify causative microorganisms by acid-fast culture examination of the resected lung tissue when granulomatous tissue is detected.

The authors state that they have no Conflict of Interest (COI).

\section{References}

1. Griffith DE, Aksamit T, Brown-Elliot BA, et al. An official ATS/ IDSA statement: diagnosis, treatment, and prevention of nontuberculous mycobacterial diseases. Am J Respir Crit Care Med 175: 367-416, 2007.

2. Sakatani M. Nontuberculous mycobacteriosis (NTM) in Japan: epidemiologic and clinical study. Kekkaku 69: 119-124, 1994 (in
Japanese).

3. Ahn CH, McLarty JW, Ahn SS, Ahn SI, Hurst GA. Diagnostic criteria for pulmonary disease caused by Mycobacterium kansasii and Mycobacterium intracellulare. Am Rev Respir Dis 125: 388391, 1982.

4. Christensen EE, Dietz GW, Ahn CH, Chapman JS, Murry RC, Hurst GA. Radiologic manifestations of pulmonary Mycobacterium kansasii infections. AJR Am J Roentgenol 131: 985-993, 1978.

5. Shitrit D, Baum GL, Priess R, et al. Pulmonary Mycobacterium kansasii infection in Israel, 1999-2004: clinical features, drug susceptibility, and outcome. Chest 129: 771-776, 2006.

6. Griffith DE, Brown-Elliot BA, Wallace RJ Jr. Thrice-weekly clarithromycin-containing regimen for treatment of Mycobcaterium kansasii lung disease: results of a preliminary study. Clin Infect Dis 37: 1178-1182, 2003.

7. Shitrit D, Priess R, Peled N, Bishara G, Shlomi D, Kramer MR. Differentiation of Mycobacterium kansasii infection from Mycobacterium tuberculosis infection: comparison of clinical features, radiological appearance, and outcome. Eur J Clin Microbiol Infect Dis 26: 679-684, 2007.

8. Kurasawa $\mathrm{T}$, Ikeda $\mathrm{T}$, Inoue $\mathrm{T}$, et al. Pulmonary infection with Mycobacterium kansasii presenting as solitary nodule shadow in the left anterior basal segment. Nihon Kyobu Shikkan Gakkai Zasshi 35: 215-219, 1997 (in Japanese).

9. Lee HS, Oh JY, Lee JH, et al. Response of pulmonary tuberculomas to anti-tuberculous treatment. Eur Respir J 23: 452-455, 2004.

10. Goo JM, Im JG, Do KH, et al. Pulmonary tuberculoma evaluated by means of FDG PET: findings in 10 cases. Radiology 216: 117121, 2000.

11. Yi CA, Lee KS, Kim EA, et al. Solitary pulmonary nodules: dynamic enhanced multi-detector row CT study and comparison with vascular endothelial growth factor and microvessel density. Radiology 233: 191-199, 2004.

12. Hosaka N, Kameko M, Nishimura H, Hosaka S. Prevalence of tuberculosis in small pulmonary nodules obtained by video-assisted thoracoscopic surgery. Respir Med 10: 238-243, 2006.

13. Gribetz AR, Damsker B, Bottone EJ, Kirschner PA, Teirstein AS. Solitary pulmonary nodules due to nontuberculous mycobacterial infection. Am J Med 70: 39-43, 1982.

14. Hahm CR, Park HY, Jeon K, et al. Solitary pulmonary nodules caused by Mycobacterium tuberculosis and Mycobcterium avium complex. Lung 188: 25-31, 2010.

15. Yonemori K, Tateishi U, Tsuta K, et al. Solitary pulmonary granuloma caused by Mycobacterium avium-intracellulare complex. Int J Tuberc Lung Dis 11: 215-221, 2007.

16. Lillington GA. Solitary circumscribed pulmonary nodule. In: A Diagnostic Approach of Chest Disease. 3rd ed. Lillington GA, Ed. Williams and Wilkins, Baltimore, 1987: 127-142.

17. Data H, Andou A, Shimizu N, et al. Evaluation of radiographic signs for the diagnosis of solitary peripheral pulmonary nodules less than $3 \mathrm{~cm}$. Acta Med Okayama 47: 53-57, 1993.

18. Hara T, Kosaka N, Suzuki T, Kudo K, Niino H. Uptake rates of ${ }^{18} \mathrm{~F}$-fluorodeoxyglucose and ${ }^{11} \mathrm{C}$-choline in lung cancer and pulmonary tuberculosis: a positron emission tomography study. Chest 124: 893-901, 2003.

19. Demura $Y$, Tsuchida $T$, Uesaka $D$, et al. Usefulness of ${ }^{18} \mathrm{~F}-$ fluorodeoxyglucose positron emission tomography for diagnosing disease activity and monitoring therapeutic response in patients with pulmonary mycobacteriosis. Eur J Nucl Med Mol Imaging 36: 632-639, 2009.

20. Kobashi Y, Mouri K, Yagi S, et al. Clinical evaluation of the QuantiFERON-TB Gold test in patients with non-tuberculous mycobacterial disease. Int J Tuberc Lung Dis 13: 1422-1426, 2009.

(C) 2011 The Japanese Society of Internal Medicine http://www.naika.or.jp/imindex.html 